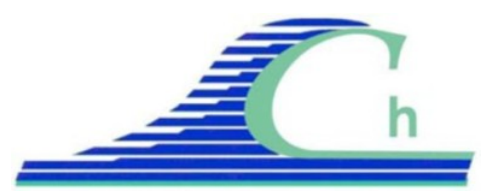

\title{
Caractérisation du transport éolien sur asphalte à partir de piégeages in situ : le cas de la digue du Braek, Dunkerque
}

\author{
Antoine TRESCA ${ }^{1}$, Marie-Hélène RUZ ${ }^{1}$, Pascal GREGOIRE ${ }^{2}$
}

\section{Laboratoire d'Océanologie et de Géosciences,}

UMR CNRS 8187 LOG - Université du Littoral Côte d'Opale, Station Marine - 28 avenue Foch, BP 80, 62930 Wimereux, France. antoine.tresca@univ-littoral.fr ; ruz@univ-littoral.fr

2. Grand Port Maritime de Dunkerque, 2505 route de l'écluse Trystram, BP 46534, 59386 Dunkerque Cedex 1, France. pgregoire@portdedunkerque.fr

\section{Résumé :}

La digue du Braek est un ouvrage de défense contre la mer du port de Dunkerque. Cette digue en enrobé bitumineux de $6 \mathrm{~km}$ de long borde une plage macrotidale orientée OSO-ENE. Le transport éolien a été évalué le long d'un profil transversal allant du haut de plage au sommet de la digue à l'aide de pièges à sédiment, pour des conditions de vent obliques. Les vitesses de vent associées ont été déterminées grâce à des anémomètres à coupelles placés à différentes hauteurs sur des mâts. Les résultats montrent une accélération du vent sur le versant de la digue exposé, similaire à l'effet de rampe observé sur les versants dunaires, ainsi que des quantités de sable piégé supérieures sur l'asphalte que sur le sable.

\section{Mots-clés :}

Transport éolien - Mesures in situ - Digue en enrobé - Nord-Pas-de-Calais

\begin{abstract}
:
The shoreline of Dunkirk Seaport partly consists of a macrotidal beach oriented WSWENE backed by a coated dike of $6 \mathrm{~km}$ long called «Digue du Braek ». Aeolian sand transport has been estimated with sand traps under different wind velocities and directions along transversal profiles on the dike. Wind velocity has been recorded with cup anemometers installed at different heights on masts. Results validate the great variability of aeolian sand transport, as it has already been observed on beaches, and show that, under oblique wind conditions, sand transport rates are more important on asphalt than on sand. Moreover, a ramp effect induced by the slope of the dike has been observed.
\end{abstract}

\section{Keywords:}

Aeolian sand transport - In situ measurements - Dike -Nord-Pas-de-Calais - France 


\section{Introduction}

La gestion des sédiments et l'entretien des ouvrages de défense contre la mer sont des enjeux d'une importance capitale pour les ports maritimes. Il faut en effet lutter contre l'érosion ou le dépôt afin de ne pas déstabiliser les infrastructures portuaires. En outre, ces infrastructures ont la plupart du temps une incidence sur les transferts de sédiments (JACKSON \& NORDSTROM, 2011) en les retenant (jetées), ou en amplifiant les processus naturels. C'est le cas de la digue du Braek, ouvrage de défense contre la mer de la façade maritime du Grand Port Maritime de Dunkerque. Cette digue en enrobé constitue une rampe d'envol des sables éoliens provenant du haut de plage et des dunes. Le sable se dépose ensuite en arrière, sur une route où environ $10000 \mathrm{~m}^{3}$ de sable ont été déblayés en 2010, ainsi que dans un bassin maritime accueillant les minéraliers, nécessitant des dragages afin de maintenir le tirant d'eau nécessaire. Des mesures in situ ont été menées afin de quantifier le transport éolien sur l'asphalte de la digue afin de pouvoir mieux appréhender les modalités de transfert de sable de la plage vers le revers de la digue. La quantification du transport éolien de sable a été étudiée à de nombreuses reprises en milieu micro à mésotidal (ARENS, 1996; BAUER et al., 1996 ; DAVIDSON-ARNOTT \& BAUER, 2009), ainsi que sur les plages macrotidales du nord de la France (MEUR-FEREC \& RUZ, 2002; RUZ \& MEUR-FEREC, 2004 ; ANTHONY et al., 2009). Cependant, très peu d'études ont été menées sur les ouvrages portuaires. Une meilleure connaissance des processus et quantités de sable transitant sur la digue permettra de trouver les meilleures solutions pour prévenir leur dépôt sur les infrastructures.

\section{Zone d'étude}

Localisée à quelques kilomètres de la frontière franco-belge, la façade maritime du port de Dunkerque, longue de $13 \mathrm{~km}$, est constituée en sa partie Est par la digue du Braek, structure en enrobé bitumineux de $6 \mathrm{~km}$ de long, connectée à une jetée de $750 \mathrm{~m}$ de long (jetée de Saint-Pol) (figure 1). Orientée OSO-ENE, la digue du Braek borde une plage macrotidale au marnage de $5,45 \mathrm{~m}$ en période de vives-eaux (SHOM), dont la granulométrie des sédiments de surface varie de 0,25 à $0,30 \mathrm{~mm}$. Elle est soumise à un régime de vents dominants longitudinaux d'origine OSO, et de façon secondaire à des vents de direction NE (figure 1). La morphologie typique à barres et à bâches de l'estran contribue à augmenter la variabilité et l'intermittence du transport éolien, puisque le sable mis en circulation sur les barres sèches, peut être rapidement capté par les bâches humides (ANTHONY et al., 2009). La jetée de St-Pol constitue un obstacle au transit sédimentaire dominant orienté vers la Belgique, et piège ainsi une quantité importante de sable à l'origine d'une progradation du trait de côte et d'un développement dunaire en pied de digue. Des dunes se sont également formées en bordure du bassin maritime, en arrière de la digue, en raison de l'accumulation depuis des années de sable du haut de plage et du pied de digue transporté par le vent (figure 1). 


\section{XII ${ }^{\text {èmes }}$ Journées Nationales Génie Côtier - Génie Civil \\ Cherbourg, 12-14 juin 2012}

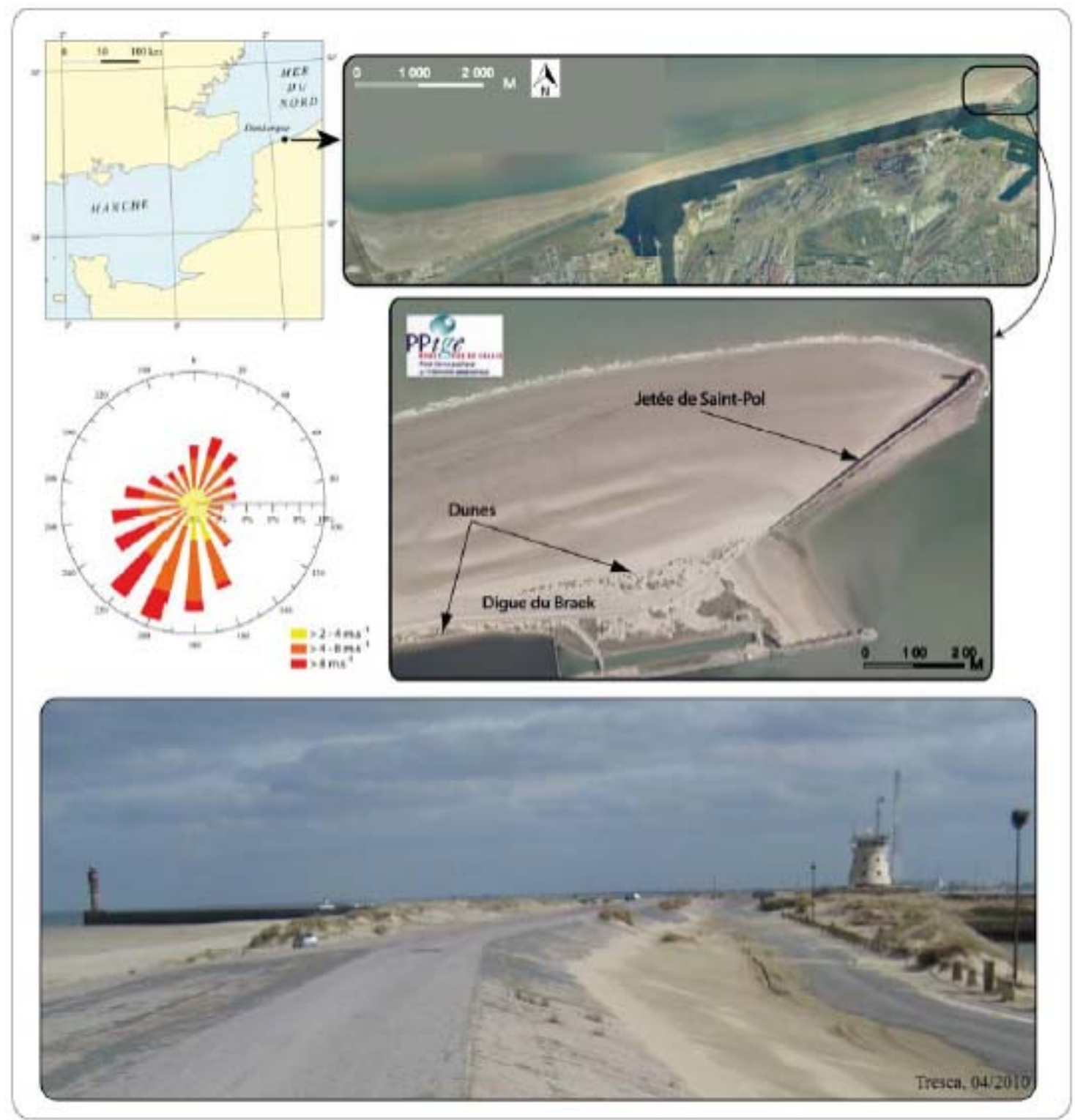

Figure 1. Localisation du site d'étude et régime des vents (1981-1990 Météo France)

\section{Matériel et méthodes}

La quantification du transport éolien s'est faite in situ à l'aide de pièges à sédiments verticaux de type GTW (NICKLING \& MCKENNA NEUMAN, 1997) (figure 2B), dont l'efficacité, testée en soufflerie par SHAO et al. (1993), est comprise entre 83 et $90 \%$ pour des vents de $9 \mathrm{~m} \mathrm{~s}^{-1}$ à $12 \mathrm{~m} \mathrm{~s}^{-1}$. Ce type de piège de forme triangulaire comporte une ouverture unidirectionnelle face au vent de $20 \times 500 \mathrm{~mm}$ (figure 2). L'arrière est fermé par une toile de $63 \mu \mathrm{m}$ collée sur les contours, permettant de retenir le sable à l'intérieur du piège. Etant donnée la légèreté de ces pièges et afin de les adapter à l'asphalte, ils sont vissés sur des plaques en fonte d'une épaisseur de $5 \mathrm{~mm}$ posées sur la digue au début de chaque séance de piégeage. L'absence de réservoir leur 
donne une capacité de stockage restreinte, limitant à 10 minutes le temps des piégeages. Les quantités de sable obtenues sont séchées à l'étuve puis converties en $\mathrm{kg} \mathrm{m}^{-1} \mathrm{~h}^{-1}$. Lors de chaque sortie, 2 ou 3 pièges ont été disposés le long d'un profil transversal, soit sur le revers, soit sur le front de digue (figure 2A). A côté de chaque piège, les vitesses de vent sont enregistrées grâce à 4 anémomètres à coupelles de type A100R (Vector Instruments) placés sur des mâts à des hauteurs de $0,25,0,5,1$ et $2 \mathrm{~m}$, ou $0,25,0,5,0,75$ et $1 \mathrm{~m}$ selon les expérimentations. Chaque anémomètre est relié par câble à un data logger, permettant d'effectuer des enregistrements des 12 anémomètres simultanément à une fréquence de 10 secondes. Les profils verticaux de vitesse du vent ont été obtenus en utilisant les données de vent moyen enregistrées pendant les 10 minutes de piégeage. La direction générale du vent est issue d'une station Deolia 96 à $24 \mathrm{~m}$ de hauteur située au sein de l'avant-port Ouest à $10 \mathrm{~km}$ du site d'étude. L'humidité du sable sur le haut de plage a été estimée en y prélevant un échantillon de surface qui a été pesé avant et après séchage à l'étuve.

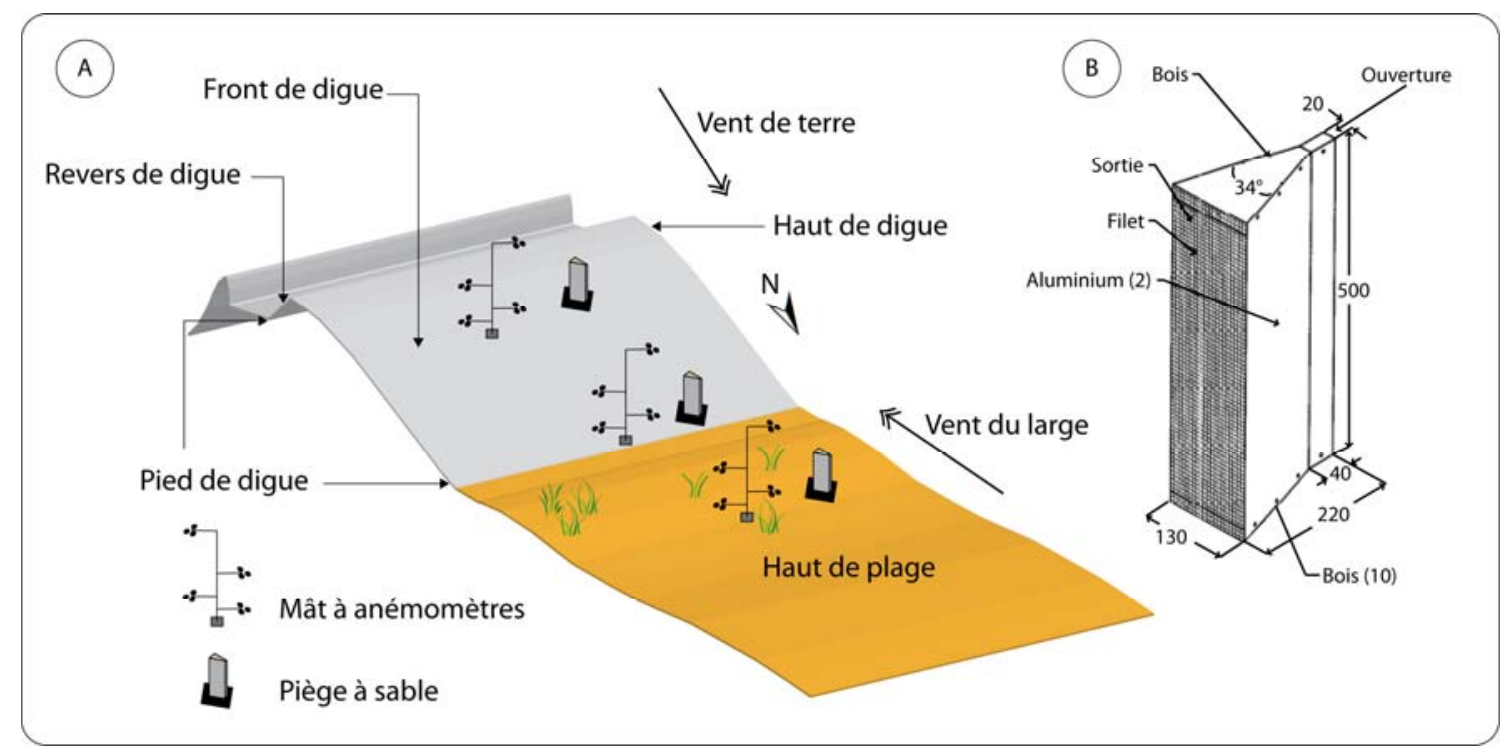

Figure 2. (A) Exemple de répartition des pièges et des anémomètres le long d'un profil transversal sur le front de digue et (B) schéma détaillé des pièges à sable utilisés (cotes en mm) (modifié, d'après NICKLING \& MCKENNA NEUMAN, 1997)

\section{Résultats}

Dix-sept séances de piégeages in situ ont été effectuées entre février et mai 2011, pour des vitesses moyennes de vent comprises entre 6 et $13 \mathrm{~m} \mathrm{~s}^{-1}$ et des valeurs d'humidité toujours très faibles, inférieures à $1 \%$ dans la grande majorité des cas. Six sorties ont été effectuées par vents de mer frontaux de direction NNO à NNE, cinq sorties par vent oblique de NE, et six lors de conditions de vent longitudinal de SSO. Les quantités de sable piégé varient de 6 à $95 \mathrm{~kg} \mathrm{~m}^{-1} \mathrm{~h}^{-1}$. Une grande variabilité caractérise les résultats 


\section{XII ${ }^{\text {èmes }}$ Journées Nationales Génie Côtier - Génie Civil \\ Cherbourg, 12-14 juin 2012}

obtenus, que ce soit sur la digue ou sur le haut de plage, comme cela a déjà pu être constaté sur les plages de la région (RUZ \& MEUR-FEREC, 2004 ; ANTHONY et al., 2009). Deux expérimentations in situ représentatives des résultats obtenus sont présentées ici. Lors de l'expérimentation du 03 mars 2011, trois pièges et trois mâts ont été disposés sur le versant de la digue exposé au vent et en haut de plage, pour des conditions de vent de NE, obliques par rapport à l'orientation du trait de côte (figure 3AIII).

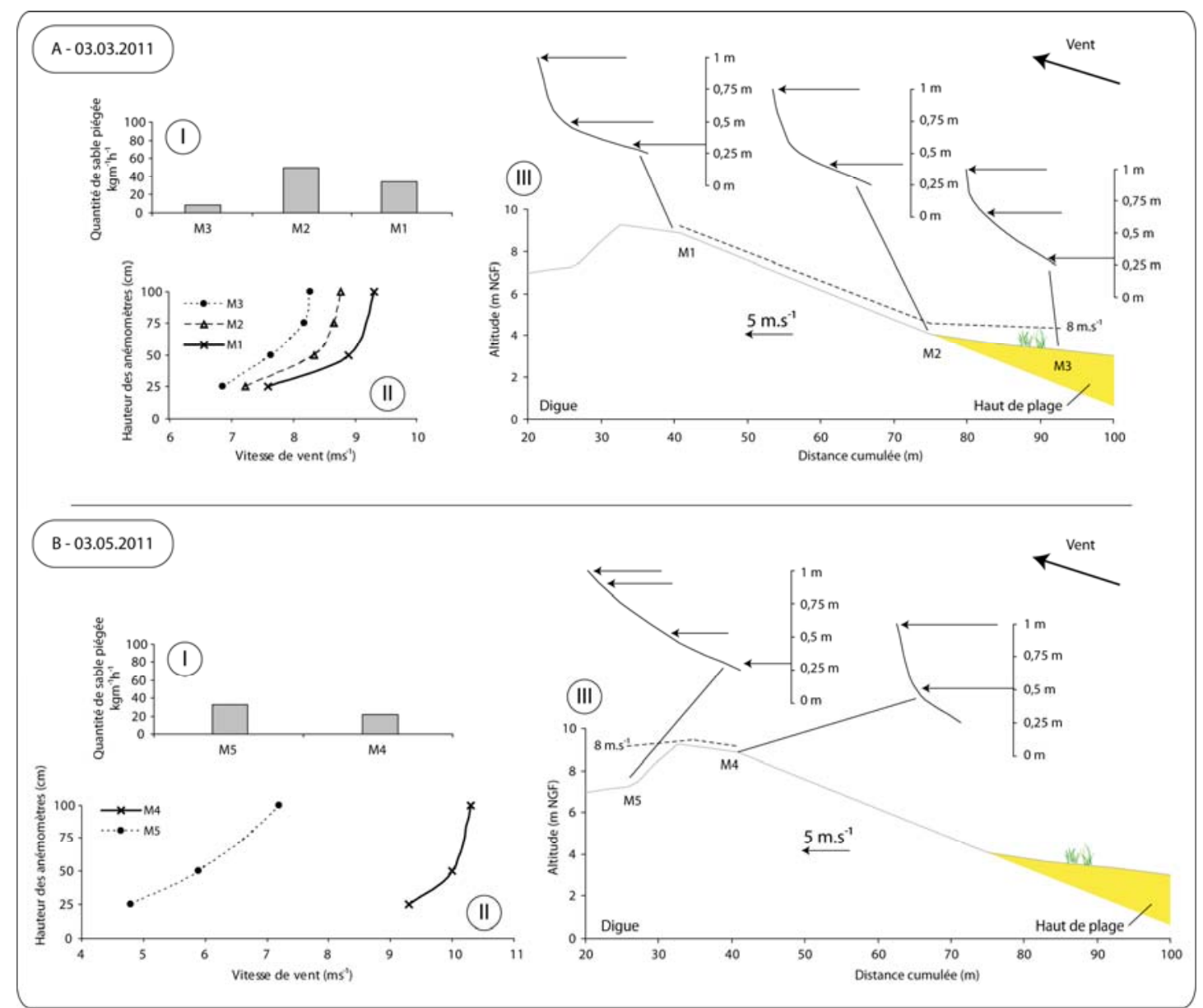

Figure 3. Profils de vitesse et quantités de sable capté le long d'un profil transversal lors de deux expérimentations du 03 mars 2011 (A) et du 03 mai 2011 (B) par conditions de vents de NE.

La vitesse moyenne du vent à $2 \mathrm{~m}$ était de $9,8 \mathrm{~m} \mathrm{~s}^{-1}$, pour un maximum de $11,3 \mathrm{~m} \mathrm{~s}^{-1}$. La limite de mise en mouvement, pour un sable de diamètre moyen de 0,275 mm (échantillon prélevé sur le haut de plage au niveau de M3 (figure 3AIII)) a été estimée à 4,7 $\mathrm{m} \mathrm{s}^{-1}$ en utilisant l'équation de BAGNOLD (1941). En prenant en compte l'humidité du sédiment, déterminée à partir de ce même échantillon à $0,4 \%$, la vitesse limite de mise en mouvement du sable a été établie à $7,3 \mathrm{~m} \mathrm{~s}^{-1}$ à l'aide de l'équation de BELLY 
(1964). Les profils verticaux de vitesse du vent ont une allure similaire au niveau des 3 mâts (figure 3AII). On constate une accélération de la vitesse du vent du haut de plage vers le sommet de digue. A $0,25 \mathrm{~m}$, on passe de $6,8 \mathrm{~m} \mathrm{~s}^{-1}$ au niveau de $\mathrm{M} 3$ à 7,6 $\mathrm{m} \mathrm{s}^{-1}$ au niveau de $\mathrm{M} 1$, tandis qu'à $1 \mathrm{~m}$ de hauteur, on passe de $8,3 \mathrm{~m} \mathrm{~s}^{-1}$ à $9,3 \mathrm{~m} \mathrm{~s}^{-1}$ de $\mathrm{M} 3$ à M1, soit une augmentation de $1 \mathrm{~m} \mathrm{~s}^{-1}$. De même, au niveau de $\mathrm{M} 2$ les vitesses mesurées à $0,25 \mathrm{~m}$ et $1 \mathrm{~m}$ sont respectivement supérieures de $0,4 \mathrm{~m} \mathrm{~s}^{-1}$ et $0,5 \mathrm{~m} \mathrm{~s}^{-1}$ par rapport aux mêmes hauteurs sur M3 situé en haut de plage. La forme des profils de vitesse varie de la même manière au niveau des trois mâts, avec une pente plus forte au dessus de 0,75 $\mathrm{m}$, ce qui indique une augmentation de la vitesse de cisaillement près de la surface topographique. Cet effet est davantage prononcé au niveau de M1 situé en sommet de digue, ce qui est interprété comme une compression des filets d'air près de la surface à ce niveau. De nombreux travaux menés sur les versants dunaires ont mis en évidence cet effet de rampe, c'est-à-dire une accélération de la vitesse du vent sur le versant exposé au vent (SVASEK \& TERWINDT, 1974 ; CHRISTIANSEN \& DAVIDSONARNOTT, 2004 ; HESP et al., 2005 ; WALKER et al., 2009). La digue joue donc ici le même rôle.

Lors de l'expérimentation du 03 mai 2011, un mât et un piège ont été déployés au niveau de la crête de digue, ainsi qu'au pied du revers, en situation d'abri (figure 3BIII). Les conditions de vent étaient similaires à celles du 03 mars 2011, de direction NE. La vitesse moyenne du vent à $2 \mathrm{~m}$ était de $10,7 \mathrm{~m} \mathrm{~s}^{-1}$ sur la crête (M4), et $8,6 \mathrm{~m} \mathrm{~s}^{-1}$ sur le revers (M5). On observe une forte différence entre les vitesses enregistrées en sommet de digue et à sa base (figure 3BII). Au niveau de M4, la vitesse atteinte à $1 \mathrm{~m}$ est de $10,3 \mathrm{~m} \mathrm{~s}^{-1}$, et elle est de seulement 7,2 $\mathrm{m} \mathrm{s}^{-1}$ au niveau de M5 à la même hauteur, soit une différence de $3,1 \mathrm{~m} \mathrm{~s}^{-1}$ entre les deux mâts. Une nette décélération du vent se produit sur le revers de la digue favorisant ainsi le dépôt du sable à cet endroit. La quantité de sable piégée est de $22 \mathrm{~kg} \mathrm{~m}^{-1} \mathrm{~h}^{-1}$ au niveau de M4, alors qu'en M5, elle est de $32 \mathrm{~kg} \mathrm{~m}^{-1} \mathrm{~h}^{-1}$ (figure 3BI). En ce qui concerne les quantités de sables captées sur le versant externe de la digue le 03 mars 2011, on observe également un dépôt important au niveau de $\mathrm{M} 2$, où la quantité de sable piégé est de $50 \mathrm{~kg} \mathrm{~m}^{-1} \mathrm{~h}^{-1}$, alors qu'elle est de seulement $8 \mathrm{~kg} \mathrm{~m}^{-1} \mathrm{~h}^{-1}$ au niveau de $\mathrm{M} 3$, soit un rapport de 1 à 6 environ (figure 3AI). La quantité de sable captée au niveau du mât M1 sur la partie supérieure de la digue est également supérieure à celle obtenue sur le haut de plage, avec $34 \mathrm{~kg} \mathrm{~m}^{-1} \mathrm{~h}^{-1}$. Par ces conditions de vents obliques de NE, l'estran alimente le piège situé en M3 sur le haut de plage, tandis que la source du sable piégé en M1 et M2 provient des dunes qui se sont formées contre la jetée de St-Pol, en pied de digue (figure 4). Ces dunes sont peu végétalisées et constituent donc un stock de sable facilement mobilisable par le vent, contrairement au sable du haut de plage où l'humidité et les laisses de mer constituent autant de freins pour le transport éolien (BAUER et al., 1996). De plus, il a été démontré que sur ces plages à barres et à bâches, le transport éolien était segmenté voire interrompu par la présence de bâches humides (ANTHONY et al., 2009). 


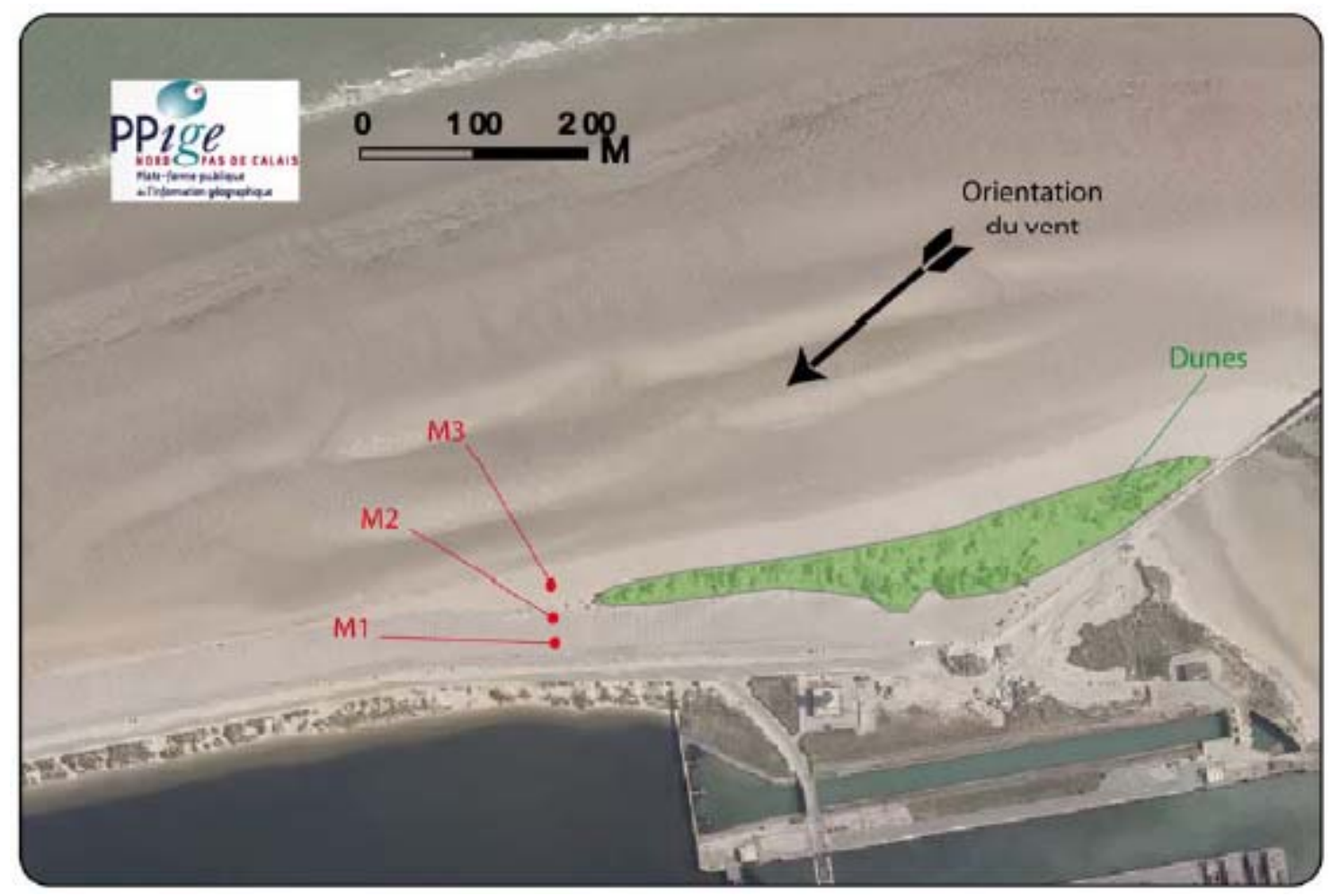

Figure 4. Vue en plan de la localisation des pièges et des dunes, ainsi que de l'orientation du vent lors de l'expérimentation du 03.03.2011.

\section{Conclusion}

Les éléments recueillis lors des mesures in situ sur la digue du Braek ont permis d'observer que les quantités de sable qui transitent sur la digue peuvent être bien plus importantes que celles qui transitent sur le haut de plage, en raison principalement de la présence de dunes peu stabilisées en pied de digue, qui constituent, lors de coups de vent de NE, une source importante de sable mobilisable, que l'on retrouve ensuite en arrière de l'ouvrage, sur la route et dans le bassin maritime. En outre, les profils verticaux de vitesse du vent ont permis de mettre en évidence un effet de rampe sur le versant exposé de la digue, similaire à celui qui a pu être observé à plusieurs reprises sur les versants dunaires. En situation d'abri, c'est-à-dire en revers de digue par vents de $\mathrm{NE}$, la vitesse diminue fortement, et l'on observe que le sable se dépose au niveau du revers de l'ouvrage. Ces éléments permettent de mieux comprendre les modalités de circulation du sable sur la digue, et aideront à une meilleure gestion des sédiments de cette unité littorale.

\section{Références bibliographiques}

ANTHONY E.J., RUZ M-H., VANHEE S. (2009). Aeolian sand transport over complex intertidal bar-trough beach topography. Geomorphology $\mathrm{n}^{\circ} 105$, pp 95-105. 
ARENS S.M. (1996). Rates of aeolian transport on a beach in a temperate humid climate. Geomorphology n¹7, pp 3-18. doi:10.1016/0169-555X(95)00089-N

BAGNOLD R.A. (1941). The physics of blown sand and desert dunes. Chapman and Hall (Ed.), London, 265 ,p.

BAUER B.O., DAVIDSON-ARNOTT R.G.D., NORDSTROM K.F., OLLERHEAD J., JACKSON N.L. (1996). Indeterminacy in Aeolian Sediment Transport Across Beaches. Journal of Coastal Research $n^{\circ} 12$ (3), pp 641-653.

BELLY P.Y. (1964) Sand movement by wind. US Army Corps of Engineers, Coastal Engineering Research Center, Tech. Memo., 1, Washington D.C., 38 p.

CHRISTIANSEN M.B., DAVIDSON-ARNOTT R.G.D. (2004). Rates of landward sand transport over the foredune at Skallingen, Denmark and the role of dune ramp. Danish Journal of Geography n ${ }^{\circ} 104$ (1), pp 31-43.

DAVIDSON-ARNOTT R.G.D., BAUER B.O. (2009). Aeolian sediment transport on a beach: Thresholds, intermittency, and high frequency variability. Geomorphology $\mathrm{n}^{\circ}$ 105, pp 117-126. doi:10.1016/j.geomorph.2008.02.018

HESP P.A., DAVIDSON-ARNOTT R.G.D., WALKER I.J., OLLERHEAD J. (2005). Flow dynamics over a foredune at Prince Edward Island, Canada. Geomorphology $n^{\circ}$ 65, pp 71-84. doi:10.1016/j.geomorph.2004.08.001

JACKSON N.L., NORDSTROM K.F. (2011). Aeolian sediment transport and landforms in managed coastal systems: a review. Aeolian research $\mathrm{n}^{\circ} 3$ (2), pp 181-196.

MEUR-FEREC C., RUZ, M-H. (2002). Transports éoliens réels et théoriques en haut de plage et sommet de dune (Wissant, Pas-de-Calais, France). Géomorphologie ${ }^{\circ} 4$, pp 321-334.

NICKLING W.G., MCKENNA NEUMAN C. (1997). Wind tunnel evaluation of a wedge-shaped Aeolian sediment trap. Geomorphology $\mathrm{n}^{\circ}$ 18, pp 333-345. doi:10.1016/S0169-555X(96)00040-2

RUZ M-H., MEUR-FEREC C. (2004). Influence of high water levels on aeolian sand transport: upper beach/dune evolution on macrotidal coast, Wissant Bay, northern France. Geomorphology n ${ }^{\circ}$ 60, pp 73-87. doi:10.1016/j.geomorph.2003.07.011

SHAO Y., MCTAINSH G.H., LEYS J.F. (1993). Efficiencies of sediment samplers for wind erosion measurements. Aust. J. Soil Res. n 31, pp 519-532. doi:10.1071/SR9930519 SVASEK J.N., TERWINDT J.H.J. (1974). Measurements of sand transport by wind on a natural beach. Sedimentology n ${ }^{\circ}$ 21, pp 311-322. doi:10.1111/j.1365-3091.1974.tb02061.x

WALKER I.J., DAVIDSON-ARNOTT R.G.D., HESP P.A., BAUER B.O., OLLERHEAD J. (2009). Mean flow and turbulence responses in airflow over foredunes: new insights from recent research. Journal of Coastal Research SI $n^{\circ} 56$, pp 366-370. 